\title{
Subtipos histológicos de liposarcoma: presentación de cuatro casos
}

\author{
M. C. CALLEJA SUBIRÁN, F. J. HERNÁNDEZ GUTIÉRREZ, C. LÓPEZ \\ ELZAURDIA, R. REVESTIDO GARCÍA \\ Servicios de Medicina Interna. Cirugía y Anatomía Patológica. Hospital Nuestra Señora \\ de Sonsoles. Ávila
}

LIPOSARCOMA HISTOLOGIC SUBTYPES: FOUR CASES REPORTS

\begin{abstract}
RESUMEN
El liposarcoma es un tumor maligno de origen mesodermico derivado del tejido adiposo. Los tipos de liposarcoma según su diagnóstico histológico son: mixoide, pleomórfico, bien diferenciado y desdiferenciado. Puede llegar a alcanzar enormes proporciones, sobre todo cuando se localiza a nivel abdominal. Su tratamiento es la cirugía radical, si es posible, junto con radioterapia y/o quimioterapia. Se presentan cuatro pacientes diagnosticados de liposarcoma, un caso de liposarcoma bien diferenciado, otro caso de liposarcoma pleomórfico y dos casos de liposarcomas mixoides; con la característica de que uno de estos presento una recidiva local con una desdiferenciación del mismo. Los cuatro evolucionaron de forma diferente. Así su pronóstico va a depender de su variedad histopatológica y de una posible multicentricidad que dificulta la exéresis quirúrgica radical completa.
\end{abstract}

PALABRAS CLAVE: Liposarcoma. Bien diferenciado. Mixoide. Pleomórfico y desdiferenciado.

\begin{abstract}
The liposarcoma is a malignant tumor of mesodermic origin derived of the adipose tissue. Liposarcoma's types, according to his histological diagnosis, are: mixoide, pleomorphic, well differentiated and dedifferentiated. It can get to reach enormous proportions, mainly when it is located at abdominal level. His treatment is the radical surgery, it is possible, together with radiation therapy and/or chemotherapy. Four patients diagnosed of liposarcoma are shown up, a case of liposarcoma well differentiated, another case of liposarcoma pleomorphic and two cases about liposarcoma mixoide; with the characteristic that one of these two cases presented a local recidivation with a dediferenciation of itself. The evolution of the four cases, was in a different way. So, their prognosis is going to depend on their histopathologic variety and on a possible multicentricidad that is going to difficult the complete radical surgical exeresis.
\end{abstract}

KEY WORDS: Liposarcoma. Well-differentiated. Myxoid. Pleomorphic and dedifferentiated.

\section{INTRODUCCIÓN}

Los sarcomas son neoplasias mesenquimatosas malignas infrecuentes $(<1 \%$ de todos los tumores malignos) que se originan en hueso y tejidos blandos. Se conocen unas 20 clases distintas que se identifican por el patrón de diferenciación hacia el tejido normal. Los sarcomas de tejidos blandos constituyen el 0,7-0,9\% (1) de todos los cánceres que aparecen en la población general. Afectan a todos los grupos de edad, pero con una edad media aproximada de 45-50 años (1-3). No existen diferencias raciales y es algo más predominante entre varones (3). Liposarcoma es la variedad histopatológica más frecuente de los tumores mesenquimatosos ya que por si mismos suponen el 0,07$0,2 \%$ de todas las neoplasias $(4,5)$.

Los tipos de liposarcoma según el diagnóstico histológico son: mixoide, pleomórfico, desdiferenciado y bien diferencia- do $(5,6)$; este ultimo es uno de los más frecuentes y el de mejor pronóstico por su escasa tendencia a producir metástasis a distancia, aunque muestra una alta incidencia de recurrencia local y, cuando esto ocurre, puede presentar un fenómeno de desdiferenciación, aumentando su agresividad (4). Presentamos cuatro casos clínicos, uno de los cuales sí que desarrollo este fenómeno de desdiferenciación.

\section{CASOS APORTADOS}

Caso 1. Varón de 60 años con antecedentes personales de EPOC moderado, que consulta por incremento del perímetro abdominal durante los meses previos sin otra sintomatología acompañante. En las pruebas complementarias solicitadas el hemograma y coagulación son normales. Glucosa, urea, ácido úrico, creatinina, calcio total, fósforo, triglicéridos, colesterol LDL, bilirrubina total, GOT, GPT, GGT, LDH, fosfatasa alcalina, amilasa, proteínas totales, albúmina, sodio, potasio, 
cloro, ferritina, transferrina, vitamina $\mathrm{B}_{12} \mathrm{y}$ folato normales. Colesterol total $243 \mathrm{mg} / \mathrm{dl}$ (110-200), colesterol HDL $120 \mathrm{mg} / \mathrm{dl}$ (42-68), hierro 44 microg/dl (65-165); Ca 19,9, 82,03 U/l (< 40). CEA, PSA y alfa-fetoproteína normales. Velocidad de sedimentación globular $41 \mathrm{~mm}(<14)$. En una segunda determinación de marcadores tumorales Ca 19,9, 91,23 y el resto normales. Resonancia magnética (RM) abdomino-pelvica: presencia de una gran masa multilobulada abdomino pelvica, cuya señal a excepción de los tabiques es preferentemente grasa. Las dimensiones máximas de la masa son de $26 \mathrm{~cm}$ en el eje cráneo caudal, 24,5 $\mathrm{cm}$ en el eje transverso y de $21 \mathrm{~cm}$ en el eje anteroposterior. Cruza la línea media y produce importante efecto de masa sobre el riñón izquierdo, al que rota y desplaza cranealmente, sin observarse signos de uropatía obstructiva. También ejerce efecto de masa sobre el colon transverso izquierdo, desplazándolo contra la pared abdominal. Los hallazgos son muy sugerentes de liposarcoma. Se realizó intervención quirúrgica mediante laparotomía media suprainfraumbilical donde se encontró una gran masa de aspecto lipomatoso desde la celda renal izquierda hasta espacio retrovesical. Se intentó extirpación en bloque con resección de fibras del psoas izquierdo infiltrado.

El informe anatomopatológico de la pieza extraída fue en su descripción macroscópica: Pieza de 45 x 40 x 15 cm. con un peso de 6.300 gr. A la superficie presenta una coloración amarillenta, y a la sección se observa que mantiene esta coloración con finos tractos conectivos, sin apreciarse de manera clara áreas de necrosis y sí una distribución en lóbulos. En la descripción microscópica las secciones histológicas muestran todas ellas un mismo aspecto: en su mayor parte están constituidas por tejido adiposo con células adiposas con tamaño grande de vacuola grande y aspecto maduro. Se observa también la presencia de núcleos atípicos de manera focal, con citoplasma vacuolado. Estas zonas de tejido maduro están distribuidas de manera difusa por toda la neoplasia, y los focos con núcleos atípicos, en ocasiones hipercromáticos se muestran de manera aislada. Diagnóstico: Liposarcoma bien diferenciado. Una nueva determinación de Ca 19,9, posterior a la intervención quirúrgica presenta normalización de la misma (24,17 U/1), manteniéndose el resto de marcadores tumorales normales. Posteriormente se realiza radioterapia complementaria, presentando una evolución favorable y permaneciendo asintomático. La RM abdominopélvica de control realizada a los cinco meses de la intervención quirurgica presenta un estado postoperatorio del liposarcoma retroperitoneal, sin evidencia macroscópica de lesión. Así este paciente presentó una evolución favorable, permaneciendo asintomático en la actualidad transcurriendo más de un año desde la intervención quirúrgica.

Caso 2. Varón de 70 años con antecedentes personales de hipertensión arterial, diabetes méllitus tipo 2 insulino dependiente y etilismo crónico severo. Isquemia en miembros inferiores con estenosis del $50 \%$ de arteria iliaca izquierda; estando pendiente de la realización de bypass femoro-femoral cuando el paciente presentó; dolor e inflamación en la zona del tríceps sural en la extremidad inferior derecha de 10 días de evolución. A la exploración, tumefacción, dolor y aumento del diámetro a nivel de la pantorrilla derecha en más de $3 \mathrm{~cm}$ respecto a la izquierda, con empastamiento difuso de dicha masa gemelar sin edema ni compromiso neurovascular. En los estudios complementarios: hemograma: normal al inicio al inicio de la enfermedad y posteriormente anemia normocítica normocrómica precisando transfusión de concentrados de hematíes en varias ocasiones. Bioquímica: glucosa: $134 \mathrm{mg} / \mathrm{dl}$ (76-120), urea $56 \mathrm{mg} / \mathrm{dl}$ (10$50)$, creatinina $1,7 \mathrm{mg} / \mathrm{dl}(0,6-1,2)$, ácido úrico $10,9 \mathrm{mg} / \mathrm{dl}(3,4-7)$ GGT 66 U/l $(<50)$, Hierro 20 microgramo/dl (65-165), Proteínas totales $5 \mathrm{~g} / \mathrm{dl}(6,2-8,8)$, albúmina $2,7 \mathrm{~g} / \mathrm{dl}(3,2-4,8)$, calcio total, fósforo, colesterol total, colesterol HDL, LDL, triglicéridos, magnesio, bilirrubina total, GOT, GPT, GGT, CK, fosfatasa alcalina, amilasa, sodio, potasio y cloro normales. $\mathrm{LDH}$ al inicio valores normales que se fueron incrementando progresivamente hasta $1811 \mathrm{U} / 1$ (120-460). PSA: 10,27 ng/ml (<4). Enolasa Especifica Neuronal (NSE) suero: $18,6 \mathrm{ng} / \mathrm{ml}$ (hasta 12,5). CEA y Ca 12,5 normales repetidamente.

Radiografía simple de extremidad inferior derecha (Fig. 1): erosión de la pared media del peroné que está en relación con la tumoración que presenta a nivel de la pantorrilla.

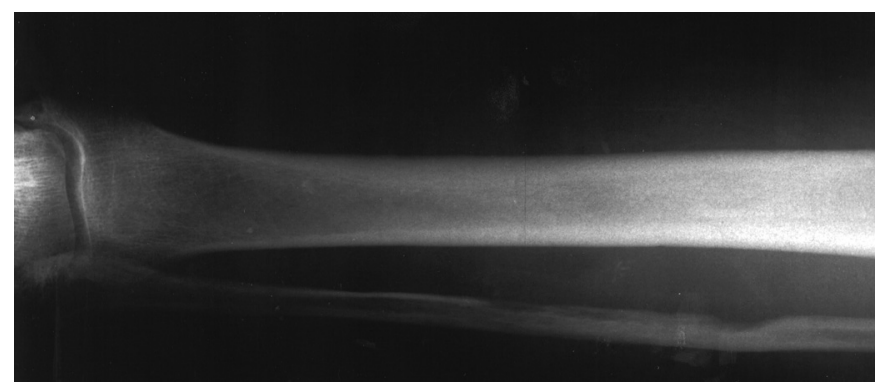

Fig. 1. Radiografía simple de extremidad inferior derecha: Erosión de la pared media del peroné que está en relación con la tumoración que presenta a nivel de la pantorrilla.

RM de pierna derecha: Gran masa de $16 \times 6 \times 7 \mathrm{~cm}$ de diámetro cráneo caudal transversal y anteroposterior respectivamente que se localiza en la región posterior de la pierna derecha, en la musculatura solea y en el tercio proximal del gemelo externo, es heterogénea, fundamentalmente isointensa con la musculatura en T1, pero mostrando áreas de hiperseñal en T1 que no se cancela en la secuencia IR, siendo muy hiperintensa en T2. Lesión sugerente de sarcoma.

Por otro lado el paciente presenta prostatismo evolutivo con polaquiuria nocturna y disuria; se solicita coincidente en el tiempo con la patología de la extremidad inferior derecha una Ecografía abdominal vesico-prostática: En zona anterior a aorta abdominal y extendiéndose desde epigastrio, hipocondrio derecho, mesogastrio hasta hipogastrio y vacío derecho se visualizan masas abdominales, sólidas con ecoestructura compleja por áreas ecogénicas, hiperecogénicas, anecoicas, voluminosas (mayor de 14,84 cm) y desde hipocondrio izquierdo ocupando vacío izquierdo se visualiza otra gran masa abdominal, sólida con ecoestructura mixta y de $13,84 \mathrm{~cm}$; que ecográficamente no se consigue determinar su origen. Próstata aumentada de tamaño, globulosa, homogénea con posibles calcificaciones. Hígado, vesícula, páncreas y riñones normales. La biopsia próstatica transrrectal no mostró malignidad. Tomografía computarizada (TC) de pelvis y abdomen: se observan grandes masas abdominales, que ocupan prácticamente toda la cavidad abdominal. Presentan una captación inhomogénea de contraste y aparentemente no guardan relación anatómica entre sí. No existen otros hallazgos patológicos significativos. TC torácica: Imágenes compatibles con bronquiectasias con discreto derrame pleural bilateral. Se realiza PAAF de una gran masa localizada a nivel de pantorrilla derecha que se encuentra toda ella marcadamente endurecida. El frotis sugestivo de neoformación mesenquimal mixoide y muestran fondo hemorrágico sobre el que se observan frecuentes grupos celulares cohesivos con patrón vascular marcado constituidos por células con núcleos monomorfos ligeramente elongados con cromatina laxa y pequeños nucleolos. Se observan citoplasmas escasos ocasionalmente microvacuolados. En la periferia de los grupos se identifica estroma metacromático con la técnica de giemsa. La celularidad neoplásica no presenta figuras de mitosis en el material obtenido. Posteriormente se procede a la intervención quirúrgica abdominal. Se extirparon en total cuatro masas una a una; son frágiles se rompen y vierten sustancia de aspecto gelatinoso; una de las cuales se encuentra en mesoileon por lo que se reseca una zona del íleon que queda sin vascularización. Anastomosis término terminal del íleon y limpieza de la cavidad. No afectación hepática. Anatomía patológica de las masas extirpadas. En la descripción macroscópica: Múltiples formaciones de aspecto nodular cuyas dimensiones son las siguientes: $14 \times 12 \times 8 \mathrm{~cm}$, otra de $19 \times 13,5 \times 5 \mathrm{~cm}$, otra de $11,5 \times$ 10 x $10 \mathrm{~cm}$, y la última $13 \times 12 \times 12 \mathrm{~cm}$. Presentan a la seriación el mismo aspecto, donde alternan áreas de aspecto mixoide con otras de consistencia blanda. Conjuntamente se remite un segmento de intestino delgado que mide $15 \mathrm{~cm}$. de longitud y que a la apertura no presenta alteraciones significativas. Descripción microscópica: Las secciones histológicas de las formaciones nodulares remitidas muestran una neoplasia mesenquimal constituida por elementos celulares de tamaño mediano, con núcleo grande y nucleolo prominente. El citoplasma es 
cantidad variable y en numerosas ocasiones con vacuola grasa. De manera frecuente se observan células que tienen un tamaño grande con una morfología estrellada y un citoplásma de aspecto eosinófilo con núcleos grandes. Las mitosis son muy escasas (menos de 5 x 10 campos de gran aumento) y se observan también amplias áreas de necrosis. También de manera focal se observan áreas mixoides. El segmento de intestino delgado remitido muestra una serosa con áreas de fibrosis; el resto de la estructura parietal no presenta alteraciones significativas. El diagnóstico anatomopatológico es de tumor mesenquimal maligno altamente sugestivo de liposarcoma mixoide. Posteriormente a la intervención quirúrgica se inicia tratamiento quimioterápico con adriamicina, con buena respuesta tumoral y clínica. Al mes de la intervención quirúrgica en el TC Abdominal de control solicitado se observan cambios postquirúrgicos con suturas metálicas de cirugía, no visualizándose imágenes de masa ni recidiva tumoral. En los dos meses siguientes presenta una progresión de su tumoración abdominal a pesar del tratamiento quimioterápico realizado con un deterioro general, disnea, incremento de sus dolores, disminución del apetito, cansancio aumento del perímetro abdominal con ascitis. A la exploración: abdomen muy distendido, duro, con gran tumoración que ocupa prácticamente todo el hemiabdomen izquierdo, donde se objetivan zonas abollonadas de distinta consistencia que son dolorosas. Edema con fóvea en abdomen, zona lumbo-sacra y extremidades inferiores. Disminución generalizada del murmullo vesicular, sobre todo en ambas bases. Resto de exploración poco significativa. En las pruebas complementarías se objetivan: Rx simple de abdomen: hay un gran efecto de masa en el abdomen central e izquierdo que desplaza todas las asas a la derecha. TC (tórax y abdomen): se observan grandes masas abdominales, fundamentalmente en hemiabdomen izquierdo, inhomogéneas y que captan contraste de manera irregular. Se acompaña de una moderada cantidad de líquido ascítico, hallazgos compatibles con recidiva tumoral. El paciente fallece a los 8 meses de la intervención quirúrgica.

Caso 3. Mujer de 74 años que consulta por tumoración axilar derecha de $5 \mathrm{~cm}$. redonda, fija y no dolorosa. Se realizan mamografía y PAAF, con resultado negativo, por ese motivo se recomendó control evolutivo.

A los dos años en los controles sucesivos realizados este nódulo mide unos $10 \mathrm{~cm}$, bien encapsulado y con bordes bien delimitados, con diagnóstico probable de fibromiolipoma benigno. Se realizaron controles en los siguientes seis años con mamografías y PAAF donde no aparecieron signos de malignidad. A los ocho años del primer diagnóstico aparece dolor en la mama derecha, persistiendo la tumoración de 10 $\mathrm{cm}$. de consistencia dura, no adherida a piel ni a planos profundos. La inspección y palpación de ambas mamas es normal. La axila izquierda está libre. En axila derecha encontramos una tumoración de más de 10 $\mathrm{cm}$ de diámetro, de consistencia dura, no adherida a piel ni a planos profundos. Resto de exploración física normal. Analítica general, ECG y radiología torácica dentro delimites normales. Mamografías: calcificaciones de necrosis grasa en mama izquierda. Las placas localizadas del tumor existente en la axila presentan bordes nítidos y es compatible con lipoma. PAAF: Frotis obtenidos por punción en los que se observan un fondo intenso de sangre lisada, grasa y escasas células epiteliales, sin signos de malignidad. Se repiten mamografías, compatibles con lipoma y PAAF en el que no aparecen signos de malignidad. A pesar de los sucesivos controles con ausencia de signos de malignidad se decide extirpar la tumoración. Siendo el diagnóstico anatomopatológico intraoperatorio de "tumor mesenquimal probablemente maligno". En la descripción macroscópica presenta varios fragmentos de tejido que miden agrupados 3 × 2 × 2 cm.; se observa una zona indurada, de coloración amarillenta blanquecina de consistencia firme. Descripción microscópica: los fragmentos de tejido muestran áreas compatibles con un tumor mesenquimal maligno de tipo liposarcoma. Se completó la disección de la axila sin conseguir extirpar totalmente la tumoración en su totalidad por estar su origen situado sobre la escápula, bajo el músculo subescapular y llega a la articulación escápulo-humeral. Siendo el resultado anatomopatológico definitivo en su descripción macroscópica de un fragmento de tejido de $16 \times 12 \mathrm{~cm}$. que aparentemente se encuentra encapsulado menos en algunas áreas periféricas. $\mathrm{Al}$ corte parece corres- ponder a tejido adiposo que presenta algunas áreas de consistencia aumentada y color más blanquecino. En la descripción microscópica se muestran áreas constituidas por tejido adiposo en el que llama la atención la presencia de algunas células con núcleos hipercromáticos y aspecto de lipoblastos. En estas áreas no se observa mucha celularidad, pero se observan las correspondientes a las áreas de mayor densidad macroscópica, que presentan escaso componente adiposo maduro y se caracterizan por células de hábito fusiforme entre las que se observan algunas giganto-celulares, muchas de ellas con hábito de lipoblastos. Se han realizado técnicas de histoquímica habiendo sido marcadamente positivas para vimentina y positivas en un $20 \%$ de células gigantes con S-100 así como las zonas de tejido adiposo maduro. La citoqueratina, desmina, actina y mioglobina han resultado negativas. Las características histológicas y los estudios de inmunohistoquímica apoyan el diagnóstico de Liposarcoma pleomórfico.

Posteriormente se procedió a radioterapia sobre el lecho tumoral con una tolerancia aceptable. Se realiza TC torácico donde no se objetivan lesiones parenquimatosas pulmonares ni adenopatías patológicas. Gammagrafía ósea con pequeño aumento de actividad en la escápula derecha. El nuevo TC torácico de control anual es normal y la nueva gammagrafía ósea es similar a la anterior. A los dos años de la intervención quirúrgica aparece disnea y cansancio apareciendo en la radiografía de tórax un derrame pleural derecho, practicándose toracocentesis, siendo el estudio citológico sospechoso de malignidad por lo que se decide comenzar con quimioterapia. A los tres años de la intervención quirúrgica continua con dolor pleural de tipo irritativo con tos seca y disnea de esfuerzo. El brazo derecho está discretamente edematoso. En la serie metastásica aparece una adenomegalia hiliar derecha. Posteriormente empeoramiento del estado general con mayor disnea y cansancio apareciendo en la radiología de tórax una veladura de todo el pulmón derecho, visualizándose en la TC torácico a la altura del lóbulo superior derecho una masa que ocupa prácticamente todo el pulmón derecho y parece corresponder a metástasis de su tumor primitivo. Estableciéndose tratamiento paliativo.

Caso 4. Varón de 66 años con antecedentes personales de HTA que consulta por aumento progresivo del perímetro abdominal de 2 años de evolución con ligero edema en extremidades inferiores sin otra clínica acompañante Anorexia desde hace un año y medio y estreñimiento progresivo. A la exploración. Auscultación cardiaca: soplo sistólico II/VI en foco aórtico. Extrasístoles aisladas. Auscultación pulmonar: crepitantes en base derecha. Abdomen globuloso, a tensión con circulación colateral periférica. Extremidades inferiores ligeros edemas que dejan fóvea hasta rodillas. Resto de exploración física normal. Se intenta la realización de paracentesis en la que no se obtiene líquido.

En las pruebas complementarias realizadas el hemograma: Hemoglobina: 12,3 g/dl, hematocrito: 36,7\% ADE: 15,9\%. Serie blanca y plaquetaria normales. Coagulación: fibrinógeno: $510 \mathrm{mg} / \mathrm{dl}$. Resto normal. Bioquímica: glucosa 123 mg/dl. Hierro 46 g/dl (65165) urea, acido úrico, creatinina calcio total, fósforo, colesterol total, HDL, triglicéridos, bilirrubina total, GOT, GPT, Fosfatasa alcalina, GGT, LDH, Proteinas totales y albúmina normales. Marcadores tumorales: CEA, Ca 19,9 y alfa-Fetoproteina normales. Marcadores tumorales repetidamente normales. Analítica de orina de 24 horas normal. Serología de hepatitis B y C negativas. Proteinograma normal. ECG: ritmo sinusal. Radiología torácica: no se observan alteraciones. Ecografía abdominal: no líquido libre, múltiples nódulos con efecto de masa. Hígado, vesícula y riñones normales. TC abdominal (Fig. 2): numerosas masas abdominales que producen un desplazamiento en el resto de los órganos y que se localizan a nivel de peritoneo y mesenterio, la de mayor tamaño mide $20 \mathrm{~cm}$ de diámetro anteroposterior y $28 \mathrm{~cm}$. en diámetro cráneo-caudal. Presentan densidad grasa y algunas calcificaciones, como primera posibilidad diagnóstica liposarcoma. Hígado, bazo y riñones normales

Imposibilidad técnica de realizar la laparoscopia por gran cantidad de adherencias. PAAF: se realizan múltiples punciones, sin poder obtener material para el diagnostico. Se procede a la intervención quirúrgica con diagnóstico intraoperatorio sugestivo de liposarcoma mixoide. Descripción macroscópica intraoperatoria: tumor mixoide altamente sugesti- 


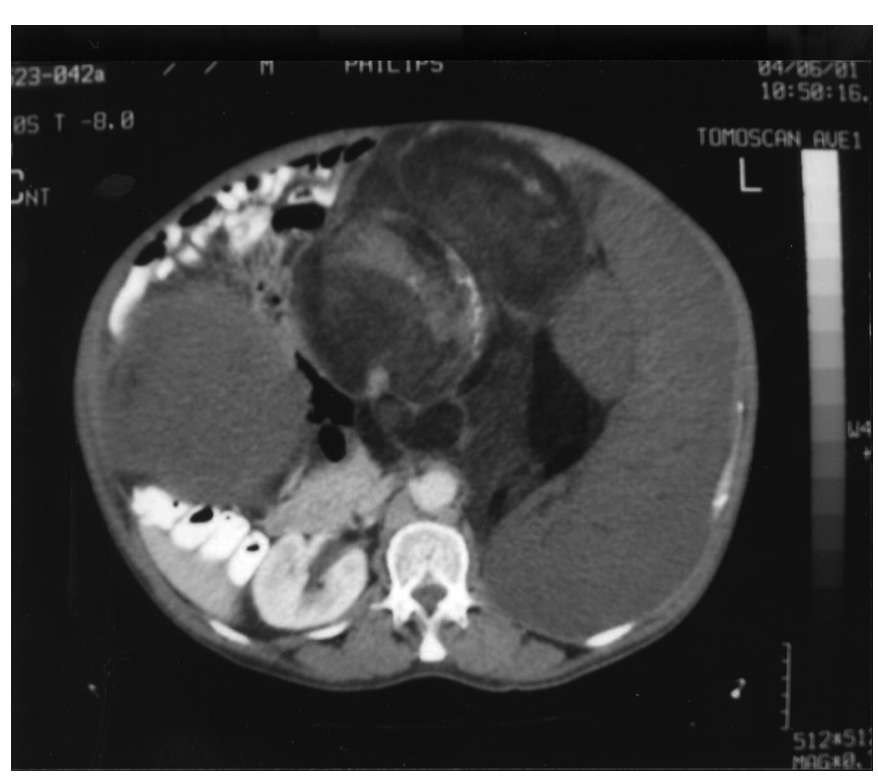

Fig. 2. Tomografía computarizada (TC) abdominal: numerosas masas abdominales que producen un desplazamiento en el resto de los órganos y que se localizan a nivel del peritoneo y mesenterio, la de mayor tamaño mide $20 \mathrm{~cm}$ de diámetro anteroposterior y $28 \mathrm{~cm}$ en diámetro cráneo-caudal; presentando densidad grasa y algunas calcificaciones.

vo de liposarcoma mixoide. El diagnóstico anatomopatológico definitivo es de liposarcoma bien diferenciado de tipo esclerosánte con áreas mixoideas y áreas de tipo pleomórfico. Segmento de intestino grueso sin alteraciones histológicas definitivas. En la descripción macroscópica presenta una masa que tiene unas dimensiones máximas de 25 × 22 x 14 $\mathrm{cm}$. que muestra una superficie lobulada bien delimitada pero no encapsulada. En los cortes seriados se identifica que está constituida en su totalidad por un tejido de superficie mucoide con áreas de aspecto amarillo y consistencia blanda. De forma aislada se observan áreas de aspecto necrohemorrágico. Posteriormente se recibe otra formación de características similares que tiene unas dimensiones máximas de $31 \times 25 \times 15$ $\mathrm{cm}$. Igualmente un pequeño fragmento irregular de tejido de $5 \times 4 \times 2$ $\mathrm{cm}$. de características similares a las dos anteriores. Se identifica un segmento de intestino grueso que tiene una longitud de 4,5 cm. Descripción microscópica muestra que está constituida por una proliferación mesenquimal adiposa con presencia de adipocitos bien diferenciados de tamaños variables junto con extensas áreas de esclerosis en las que se identifican frecuentes lipoblastos. Frecuentemente se observan áreas de matriz mixoide con patrón vascular arboriforme así como extensas zonas con presencia de células marcadamente pleomórficas de patrón estrellado frecuentemente multinucleadas, con citoplasma amplio y eosinófilo. Entre la celularidad neoplásica se observan áreas de necrosis con infiltrado de leucocitos polimorfonucleares neutrófilos. La neoformación no presenta cápsula. El segmento de intestino grueso no muestra alteraciones histológicas significativas ni evidencia de infiltración tumoral.

Posteriormente se realizó tratamiento quimioterápico y radioterapia complementaria.

A los dos meses de la intervención quirúrgica en el TC abdomen de control presenta: masa de partes blandas en la cadena iliaca externa izquierda 7 x $3 \mathrm{~cm}$ de diámetro compatible con conglomerado adenopático que provoca un desplazamiento lateral derecho de la vejiga. Posteriormente a los seis meses del primer acto quirúrgico se interviene de nuevo de una tumoración retroperitoneal izquierda que infiltra hasta sacroilíaca izquierda y engloba a la vena ilíaca. Se extirparon 3/4 de tumoración dejando tumor sobre vena iliaca y sacroiliaca izquierda; siendo el resultado anatomopatológico de los nodulos retroperitoneales en la descripción macroscópica de: fragmentos irregularmente nodulares que en conjunto presentan unas dimensiones de $18 \times 13 \times 7,5 \mathrm{~cm}$. Al corte muestran aspecto homogéneo, sólido y pardoamarillento. En su descripción microscópica aparece una neoplasia mesenquimal bifásica con predominio de áreas de sarcoma no lipogénico de alto grado, de las que emergen en continuidad, áreas de tumor lipomatoso atípico con patrón escleroso prominente. Las zonas de sarcomas no lipogénico presentan imágenes histológicas semejantes a un fibrohistiocitoma maligno, con zonas de más de 5 mitosis x 10 campos de gran aumento, ocasionalmente atípicas. Predomina la celularidad fusiforme, con numerosas células pleomórficas, multinucleadas y gigantonucleadas. En otras zonas las células presentan características fusiformes. No se ha observado diferenciación a lipoblastos entre las células mesenquimales descritas. El otro componente de la neoplasia bifásica muestra numerosas células adiposas, con estroma levemente fibroso con componente de mayor polimorfismo nuclear. La inmunoexpresión de p53 es de 5-10\% en los núcleos, con mayor proporción en las áreas de sarcoma no lipogénico. Se interpreta como signo de progresión del tumor. Puede verse un ganglio linfático en una de las zonas periféricas del tumor, infiltrado por el mismo. La neoplasia mesenquimal descrita se acompaña de componente inflamatorio moderado, entremezclado con las células neoplásicas, esto constituye una neoplasia mesenquimal de alto grado compatible con liposarcoma desdiferenciado. Se realiza tratamiento radioterápico paliativo con buena tolerancia al mismo y controles consecutivos de TC torácicos y abdominales sin alteraciones radiológicas significativas hasta que a los 3 años aparece en lóbulo medio del pulmón derecho una imagen nodular, hipodensa, ovoidea de $5 \mathrm{~cm}$ de diámetro anteroposterior y $2,5 \mathrm{~cm}$ transversal que es compatible con lesión metastático. En el TC abdominal no se objetivan adenopatías patológicas abdominales ni pélvicas, así mismo aparece una tumoración de partes blandas y de bordes mal definidos en la zona interna del hueso iliaco izquierdo que no se diferencia del músculo iliaco y desplaza medialmente a los vasos. Estos hallazgos sugieren recidiva o metástasis del liposarcoma retroperitoneal intervenido.

\section{DISCUSIÓN}

Los liposarcomas no suelen ser lipomas degenerados sino que aparecen de "novo". Generalmente están bien definidos, aunque por tener gran cantidad de estroma no suele verse la grasa tan claramente como en los lipomas. Se visualiza como una masa mal delimitada de los tejidos circundantes. Si se encuentran cerca de estructuras óseas pueden erosionarlas por presión extrínseca como sucedió en nuestro segundo caso. Presentan un tamaño medio entre $13-15 \mathrm{~cm}(2,3)$. Cuando afecta a nivel abdominal suelen ser masas grandes y pueden alcanzar enormes proporciones; presentando entonces un diámetro medio de 20-25 cm, en nuestros casos las medidas fueron incluso superiores a la media y un peso que varía entre 3 $20 \mathrm{~kg}$ (7) incluso hasta $42 \mathrm{~kg}$ en alguna ocasión (8) con compromiso de los órganos vecinos hasta en un $80 \%$ (4); como así sucedió en tres de los casos presentados.

Aproximadamente un $60 \%$ de sarcomas de tejidos blandos se originan en los miembros, siendo tres veces más frecuente en los miembros inferiores $(94 \%)(3,9)$ que en los superiores; el $30 \%$ se localiza en el tronco y un $40 \%$ de ellos son retroperitoneales.

De los cuatro tipos histológicos de liposarcoma: bien diferenciado, mixoide, pleomórfico y desdiferenciado. El liposarcoma mixoide constituye el más frecuente subtipo de liposarcoma $(1,2,9,10)$, aproximadamente $40-50 \%$ de todos. Los liposarcomas desdiferenciados pueden surgir de novo o bien como recurrencia de otro tipo de liposarcoma. Desde el punto de vista clínico la manifestación más frecuente es la aparición de una masa asintomática; puede haber síntomas mecánicos atribuibles a compresión, tracción o atrapamiento de nervios y músculos (7-9).

Cuando la localización es a nivel abdominal, la clínica suele ser un dolor difuso en esta localización acompañado de anore- 
xia y adelgazamiento, con aumento del perímetro abdominal. El signo más característico es la masa abdominal palpable indolora detectable en aproximadamente el $78 \%$ de los casos, que produce sintomatología abdominal por compresión de órganos. En varios de nuestros casos esa fue la forma de presentación siendo generalmente asintomáticos y con común denominador el aumento del perímetro abdominal como único signo.

El liposarcoma al ser poco vascularizado tiene baja tendencia a producir metástasis a distancia y su pronóstico depende de su variedad histológica y de una posible multicentricidad que dificulte la exéresis quirúrgica completa. Entre los factores pronósticos, el grado de malignidad histológica, la relación con los planos aponeuróticos y el tamaño del tumor primario constituyen los más importantes así como la diferenciación celular, el número de mitosis y la extensión de la necrosis (4).

Las metástasis a distancia después de la intervención inicial se modifican con la variedad histológica así; los liposarcomas bien diferenciados (llamados también tumores lipomatosos atípicos) carecen de poder metastático, comportamiento que siguió el caso presentado y los liposarcomas mixoides casi nuncan metastatizan, pero cuando lo hacen tienen afinidad por sitios poco habituales que contienen grasa, como el retroperitoneo $71 \%$ (10), intraabdominal extrahepático $50 \%$, espinal/paraespinal (43\%) (11), el mediastino y el tejido subcutáneo. En el liposarcoma mixoide de extremidades potencialmente la metástasis extrapulmonar es mas frecuente aunque también puede ser pulmonar (11). Otras localizaciones de metástasis menos frecuentes de LM pueden ser: cabeza, cuello y pelvis (2), escrotal (8) y paravesical. Pacientes con liposarcomas localizados primariamente en la extremidad, el incremento significativo de fracaso local fue visto en pacientes con alto grado de liposarcoma. El $50 \%$ de pacientes con liposarcoma pleomórfico tuvo recaídas o reincidencias en 5 años (12). Los liposarcomas desdiferenciados se comportan igual que otros sarcomas de gran malignidad. Las metástasis a distancia no son significativas en las formas bien encapsuladas, bien diferenciadas o en los tumores mixoides; sin embargo, alcanza tasas del $85-90 \%$ en las formas desdiferenciadas y en los tumores pleomórficos. Estas metástasis se localizan esencialmente en el pulmón, como sucedió en nuestro caso.

El liposarcoma desdiferenciado es un tipo de liposarcoma en el cual la transmisión de bajo grado a alto grado dentro del liposarcoma bien diferenciado es observado y esta usualmente ocurre de un modo abrupto; mientras que en raros casos este puede ser de forma gradual $(13,14)$. Liposarcoma mixoideo puede mostrar, aunque muy raramente, características histológicas de desdiferenciación (15). Sorprendentemente la clínica de liposarcoma desdiferenciado es menos agresiva que en los sarcomas pleomóficas de alto grado (13). El liposarcoma mixoide usualmente es una enfermedad indolente, pero hay un subtipo de pacientes que desarrollan metástasis en tejidos blandos los cuales, tienen significativamente peor pronóstico y esto puede suceder años después del diagnóstico inicial, pudiéndose asociar con un periodo de supervivencia mediolargo por lo cual debe ser manejado agresivamente, en uno de nuestro caso ya presentaba las metástasis en el momento del diagnóstico. La recidiva local es la norma si no están bien encapsulados o no son resecados totalmente. El liposarcoma pleomórfico fue altamente maligno con una recurrencia local de un $37 \%$ y metástasis en 10 años del $41 \%$. Grandes tumores pleomórficos tuvieron un porcentaje de metástasis altamente significativas en comparación con los pequeños. Estos facto- res biológicos se reflejaron en la progresiva supervivencia a los 10 años, $87 \%$ en tumores bien diferenciados, $76 \%$ en tumores mixoideos y 39\% en tumores pleomórficos (16).

La localización del liposarcoma en el retroperitoneo es un factor independiente de mal pronóstico asociado con una presentación en la edad adulta (> 50 años), con tamaños tumorales $>10 \mathrm{~cm}$, con presencia de márgenes quirúrgicos positivos y con afección multiorgánica por su tendencia a la multicentricidad. (4) El desenlace clínico del liposarcoma mixoide es mejor que otros tipos histológicos pero este es muy pobre cuando el tumor aparece de forma multifocal (7), como así se presento en uno de nuestros casos.

La radiografía simple, la ecografía, la Tomografía computarizada (TC) y la RM son válidas para su estudio; la naturaleza del tumor se sugiere por la hipodensidad característica del tejido adiposo. Sin embargo, su resolución puede verse disminuida en presencia de calcificaciones, componentes fibrosos, necrosis y hemorragia. La RM tiene una densidad superior a la de la grasa normal, pudiendo llegar a la densidad cercana a la del agua o incluso a la del músculo. Desde el punto de vista del examen por TC, la densidad de estos tumores es mezcla de grasa, músculo y agua. Los principales problemas en la TC es que algunos tumores, como un liposarcoma mixoide bien definido y esférico, puede simular en los cortes tomográficos un quiste benigno o un absceso. La RM es superior para la diferenciación de tejidos específicos adyacentes y, por tanto, para la estadificación tumoral. Las imágenes de RM de liposarcoma se correlacionan con la histología $(5,9,17)$. La tomografía por emisión de positrones ha mostrado su eficacia para la evaluación de recidivas y enfermedad metastásica. La citología por punción dirigida por ecografía o TC permite filiar la estirpe tumoral de forma preoperatorio (4) La radiografía simple y la RM obtienen las mejores imágenes cuando el tumor primario está situado en los miembros como en nuestro caso.

Se han descrito recientemente alteraciones genéticas y moleculares en los liposarcomas; las alteraciones descritas con más frecuencia son las amplificaciones en la región $12 \mathrm{q}$ 13-15 que implican a los genes MDM2, CDK4 y que tienen implicaciones no solo para establecer el diagnóstico de malignidad, sino para en el futuro delimitar mejor el pronóstico de estos tumores (4).

La cirugía conservadora y radioterapia tienen excelentes resultados locales y relativamente pequeño riesgo de metástasis para pacientes con liposarcoma bien diferenciado y mixoideo. La misma estrategia de tratamiento parece apropiada para liposarcoma pleomórfico pero con menos expectación de resultados satisfactorios (16).

Las formas bien diferenciadas y/o mixoideas parecen tener un pronóstico más favorable a largo plazo. Este pronóstico justifica las intervenciones quirúrgicas repetidas (18).

El planteamiento inicial de esta tumoración es quirúrgico con amplia resección $(1,19)$ los sarcomas de los tejidos blandos tienden a crecer a lo largo de los planos aponeuróticos buscando las líneas de menor resistencia. Debido a ello, los tejidos blandos circundantes son comprimidos y forman una pseudocápsula que presta al sarcoma el aspecto de una lesión bien encapsulada. Esto es siempre algo engañoso, ya que la simple "enucleación" o la extirpación marginal de tales lesiones es seguida de unas posibilidades de recidiva local del 50\% al $90 \%$. La extirpación radical con unos bordes negativos, incluido el sitio de donde se tomó la biopsia, es la técnica quirúrgica de elección en las formas localizadas. La radioterapia postoperato- 
ria (60-70 Gy) disminuye las tasas de recidiva local en formas no bien encapsuladas o poco diferenciadas. También parece haber acuerdo en la utilización de esta radioterapia de forma paliativa en tumores no operables o en caso de resección incompleta ya que, a pesar de que los tumores mesodérmicos son radiorresistentes, el liposarcoma es el más radiosensible. Aunque se ha apuntado que la radioterapia podría aumentar la supervivencia y el intervalo libre de enfermedad, hay otros autores para los que este tratamiento no mejora la supervivencia de la enfermedad especifica a largo plazo en casos de resecciones macroscópicamente completas (4). Así, la cirugía que respeta el miembro está indicada; excepto, cuando no pueden obtenerse unos bordes negativos de la pieza de resección, cuando el riesgo de la radioterapia es inaceptable o cuando están afectadas las estructuras vasculonerviosas de tal modo que la resección, va a tener serias consecuencias funcionales para el miembro (11). A pesar de la heterogeneidad histológica, la mayoría de los sarcomas de tejidos blandos son poco sensibles a la quimioterapia (2) siendo, el liposarcoma mixoide relativamente quimiosensible en comparación con los otros liposarcomas (6). En la fase avanzada del proceso tumoral los sarcomas de tejidos blandos con metástasis son en su mayoría incurables, pero hasta el $20 \%$ de los pacientes que entran en remisión completa logra supervivencias a largo plazo. Por tanto, los esfuerzos terapéuticos pretenden lograr una remisión completa con la cirugía, quimioterapia y radioterapia o con todos los recursos. La resección quirúrgica de las metástasis, siempre que pueda hacerse, forma parte del tratamiento, como así se realizó con nuestros pacientes. Algunos mejoran tras repetidas extirpaciones de las metástasis (2). Ante todo se debe tener un umbral bajo en la evolución inicial de síntomas menores en pacientes con liposarcoma ya que estos, pueden reflejar las primeras manifestaciones de una metástasis extrapulmonar o pulmonar para su detección temprana (20). Es recomendable realizar un seguimiento estricto mediante TC o RM cada 6 meses dado su alto porcentaje de recidivas.

\section{Bibliografía}

1. Behranwala KA, Roy P, Giblin VA,Hern R, Fisher C, Thomas JM. Intra-abdominal metastases from soft tissue sarcoma. J Surg Oncol 2004; 87: 116-20.

2. Patel SR, Burgess MA, Plager C, Papadopoulos NE, Linke KA, Benjamin RS. Myxoid liposarcoma. Experience with chemotherapy. Cancer 1994; 74: 1265-9.

3. Oliveira AM, Nascimiento AG, Okuno SH, Lloyd RV. P27 (kip1) protein expression correlates with survival in myxoid and round-cell liposarcoma. J Clin Oncol 2000; 18: 2888-93.

4. Echenique-Elizóndo M, Amondarain-Arratibel JA. Giant retroperitoneal liposarcoma 2005; 77: 293-5.

5. Uhl M, Roeren T, Schneider B, Kauffmann GW. Magnetic resonance tomography of liposarcoma. Rofo 1996; 165: 144-7.

6. Jones RL, Fisher C, Al-Muderis O, Judson IR. Differential sensitivity of liposarcoma subtypes to chemotherapy. Eur J Cancer 2005; 41: 285360.

7. Nohara T, Kawashimai A, Nagahara A, Kitamura M, Akai H, Okai T et al. Multifocal liposarcoma showing rapid growth in a short term: A case report. Hinyokika Kiyo 2005; 51: 21-3.

8. Yol S, Tavli S, Tavli L, Belviranli M, Yosunkaya A. Retroperitoneal and scrotal giant liposarcoma: report a case. Surg Today 1998; 28: 33942.

9. Soulie D, Boyer B, Lescop J, Puyol A, Le Friant G, Cordoliani YS. Myxoid liposarcoma. MRI imaging. J Radiol 1995; 76: 29-36.

10. Spillane AJ, Fisher C, Thomas JM. Myxoid liposarcoma the frequency and the natural history of nonpulmonary soft tissue metastases. Ann Surg Oncol 1999; 6: 389-94.

11. Estourgie SH, Nielsen GP, Ott MJ. Metastatic patterns of extremity myxoid liposarcoma and their outcome. J Surg Oncol 2002; 80: 89-93.

12. Chang HR, Hajdu SI, Collin C, Brennan MF. The prognostic value of histologic subtypes in primary extremity liposarcoma. Cancer 1989; 64: 1514-20.

13. Dei Tos AP. Liposarcoma: New entities and evolving concepts. Ann Diagn Pathol 2000; 4: 252-66.

14. Chorneyko K. The ultrastructure of liposarcoma with attention to "dedifferentiation". Ultrastruct Pathol 1997; 21: 545-57.

15. Mentzel T, Fletcher CD. Dedifferentiated myxoid liposarcoma: a clinicopathological study suggesting a closer relationship between myxoid and well-differentiated liposarcoma. Histopathology 1997; 30: $457-63$.

16. Zagars GK, Goswitz MS, Pollack A. Liposarcoma: Outcome and prognostic factors following conservation surgery and radiation therapy. Int J Radiat Oncol Biol Phys 1996; 36: 311-9.

17. London J, Kim EE, Wallace S, Shirkhoda A, Coan J, Evans H. MR imaging of liposarcomas: correlation of MR features and histology. J Comput Assist Tomogr 1989; 13: 832-5.

18. Boutboul R, Campan P, Josso B, Ival M, Vincenti N. Prolonged survival in retroperitoneal liposarcoma. Apropos of a case with 27 years, survival. Prognostic value of the histological type and role of repeat surgery. Ann Urol 1986; 20: 397-9.

19. Ohashi T, Imokawa H, Kenmochi M, Sato S. Neck metastasis of a myxoid liposarcoma of the lower extremity. Auris Nasus Larynx 2004; 31: 443-6.

20. Vassilopoulos PP. Voros DN, Kelessis NG, Katsilieris JN, Apostolikas NG. Unusual spread of liposarcoma. Anticancer Res 2001; 21 (2B): 1419-21. 